\title{
Effect of Land Configuration, Irrigation Level and Nutrient Management on Growth, Yield and Economics of Turmeric (Curcuma longa L.)
}

\author{
H. H. Dikey ${ }^{\text {* }}$, V. M. Bhale ${ }^{2}$, V. S. Kale ${ }^{3}$ and R. S. Wankhade ${ }^{4}$ \\ ${ }^{1}$ Regional Research Centre, Dr. Panjabrao Deshmukh Krishi Vidyapeeth, \\ Amravati-444603, Mahrashtra, India \\ ${ }^{2}$ Dr. Panjabrao Deshmukh Krishi Vidyapeeth, Akola, Mahrashtra, India \\ ${ }^{3}$ Department of Vegetable Science, Dr. Panjabrao Deshmukh Krishi Vidyapeeth, \\ Akola, Mahrashtra, India \\ ${ }^{4}$ Agriculture Research Station, Dr. Panjabrao Deshmukh Krishi Vidyapeeth, \\ Achalpur Dist. Amravati-Mahrashtra, India \\ *Corresponding author
}

\section{A B S T R A C T}

\section{Keywords}

Organic manures, Curcuma longa L., rhizome yield, CPE

\section{Article Info}

Accepted:

22 August 2019

Available Online:

10 September 2019
A field experiment was carried out to study the effect of land configuration, irrigation level and nutrient management on growth, yield and economics of turmeric (Curcuma longa L.) at the Research Farm, Department of Agronomy, Dr. Panjabrao Deshmukh Krishi Vidyapeeth, Akola (Maharashtra) during kharif season of the year 2014-15 and 2015-16. A set of twenty four treatment combinations of land configuration, irrigation level and nutrient management $(2 \times 3 \times 4)$ were laid out in a Split plot design with three replications. The experimental results revealed that, broad bed furrow land configuration, irrigation level $40 \mathrm{~mm} \mathrm{CPE}$ and application of $100 \% \mathrm{RDF}+25 \% \mathrm{RDN}$ through vermicompost recorded significantly more number of tillers, plant height, number of functional leaves, leaf area and dry matter plant- 1 of turmeric. The entire yield attributes and economics i.e. number of fresh mother rhizomes, weight of fresh mother rhizomes, and yield of fresh mother rhizomes, yield of fresh primary fingers, yield of fresh secondary fingers, total yield of turmeric ha ${ }^{-1}$ and yield of cured fingers $\mathrm{ha}^{-1}$, gross monetary returns, net monetary returns and benefit cost ratio $\mathrm{ha}^{-1}$ were recorded significantly superior in broad bed furrow of land configuration, irrigation level $40 \mathrm{~mm}$ CPE, application of $100 \% \mathrm{RDF}+25 \% \mathrm{RDN}$ through vermicompost and treatment combination of $40 \mathrm{~mm} \mathrm{CPE}$ with $100 \% \mathrm{RDF}+25 \% \mathrm{RDN}$ through vermicompost.

\section{Introduction}

Turmeric (Curcuma longa L.) is one of the second most important spice crops in foreign exchange earnings after chilli. India is the largest producer of turmeric, supplying $94 \%$ of the world's demand. India also dominates the world production scenario contributing to 
approximately $78 \%$ of world's total production producing $6,00,000$ tonnes of turmeric annually. India produced about 9.43 lakh tonnes of turmeric from 1.85 lakh hectare in year 2015-16. Turmeric occupies $6 \%$ of the total area under spices and condiments in India (Anonymous, 2015). India has been a traditional producer, consumer and exporter of turmeric, but the productivity of turmeric in India is lowest in the world.

Integrated nutrient management envisaging conjunctive use of inorganic and organic sources of nutrient is a novel system of plant nutrient use for sustaining soil health and crop productivity. The integrated nutrient management ensures the better and sustainable yield while correcting some secondary and micronutrients deficiencies and it also increases the nutrient use efficiency. Moreover, integrated nutrient management is of immense importance in high value crop like turmeric and by improving the productivity of this crop, the socio-economic status of the farmers of the state can be improved further.

Land configuration helps for maximizing rainfall infiltration, minimizing erosion, total runoff, facilitates drainage and ultimately improves water use efficiency. The raised bed zone of broad bed and furrow system is better aerated with lower penetration resistance and favourable for deeper seed placement and better crop emergence (Jayapaul et al., 1996). Suitability of different land configurations depends on soil type and rainfall pattern. Unique physical properties of heavy clay soils are the greatest limitations to the agricultural production. In spite of sufficient water and favourable weather conditions, the productivity of such soils is threaten owing to low infiltration, poor internal drainage, narrow workable moisture range, variable moisture availability, inadequate seeding emergence and loss of soil structure. Such soils require a careful management to prevent soil quality deterioration in order to tap potential production. Land configuration, which involves different methods of seed bed preparations, is one of the most important management practices which increases input use efficiency and crop production.

Water and fertilizer are the two important inputs for agricultural production and are interrelated in their effects on plant growth and yield. However, shortage of water for irrigation is being increasingly exploited due to pressures from depleting groundwater levels, rising alternative demands, water quality degradation and economics. Nutrient availability in the soil-plant system is dictated by complex interactions between plant roots, soil microorganisms, chemical reactions and pathways of losses.

Turmeric is commercially cultivated however, the production of turmeric per unit land area in vidarbha is very low because of the poor knowledge on improved cultivation technology to the farmers. Considering the all above facts, the present investigation is therefore planned to study the effect of land configuration, irrigation level and nutrient management on growth, yield and economics of turmeric.

\section{Materials and Methods}

A field experiment was carried out at the Research Farm, Department of Agronomy, Dr. Panjabrao Deshmukh Krishi Vidyapeeth, Akola (Maharashtra) during kharif season of the year 2014-15 and 2015-16. A set of twenty four treatment combinations of land configuration, irrigation level and nutrient management $(2 \times 3 \times 4)$ were laid out in a Split plot design with three replications. The Turmeric crop (variety - PDKV Waigaon) was raised using nutrient dose @ RDF 200:100:100 Kg NPK per hectare. The quantity of fertilizers to be applied were 
calculated on gross plot basis. Nitrogen, Phosphorus and Potassium were applied in the form of urea, single super phosphate and muriate of potash respectively. The fertilizers as per treatments were applied at the spot of planting in rings and were thoroughly mixed in the soil with the help of weeding hook. Vermicompost was applied to different treatment plots. The quantity required was calculated based on the results of analysis. A seed rhizome was planted at $10 \mathrm{~cm}$ depth in the center of ridge in ridges and furrow method and three lines were planted on broad bed furrow at given spacing of $45 \mathrm{~cm} \mathrm{X} 22.5$ $\mathrm{cm}$ at the rate of 25 quintal ha-1. At the time of planting mother rhizomes were treated with Carbendenzim 50 WP @ 1gm + Quinalphos 2 $\mathrm{ml} / 1$ for 30 minutes. The irrigation system of pvc pipes consisted of main and sub-main of 75, 63 and $50 \mathrm{~mm}$ diameter were laid in each plot for irrigation. Control valves were fixed on main and sub-main pipeline. Water meter was fixed on main pipeline for measuring the discharge of water during the irrigation. Measured quantity of water was applied to each plot. For irrigating the plots at different irrigation levels control valves was opened in each replication and plots were irrigated. During the year 2014-15, total 15, 10 and 08 irrigations were given to 40, 60 and $80 \mathrm{CPE}$ irrigation levels where as 19, 14 and 10 irrigations were applied to 40, 60 and $80 \mathrm{CPE}$ irrigation levels respectively in the year 201516. Irrigations were applied as per irrigation levels after planting. The depth of each irrigation was $10 \mathrm{~cm}$ in case of ridges and furrow and broad bed furrow planting which was measured with water meter which was fitted on pvc pipe line. Weedicide application, weeding, earthing up and plant protection measures were undertaken as and when required as per recommendation. The crop was harvested at full physiological maturity. Five plants in each treatment per replication were tagged randomly for recording the observations on seedling, growth and yield parameters and mean values were subjected to statistical analysis. Biometric observations recorded during the course of investigation along with sample size and recording time. Data collected during the course of investigation ware statistically analyzed by adopting standard procedure of 'Analysis of Variance' by Panse and Sukhatme (1967). The growth parameters like number of tillers plant ${ }^{-}$ ${ }^{1}$, plant height, number of functional leaves plant $^{-1}$, leaf area plant ${ }^{-1}$ and dry matter plant ${ }^{-1}$ were recorded. The data on Yield attributes, yield and economics were taken on number of mother rhizomes per plant, weight of mother rhizomes per plant, yield of fresh mother rhizomes $\mathrm{ha}^{-1}$ (q), yield of fresh primary fingers $\mathrm{ha}^{-1}(\mathrm{q})$, yield of fresh secondary fingers ha ${ }^{-1}(q)$, total yield of turmeric (Mother + Primary+ Secondary) ha ${ }^{-1}(q)$, yield of cured fingers ha ${ }^{-1}$ (q), Gross monetary returns (Rs. / ha), Net monetary returns (Rs. / ha) and B : C ratio.

\section{Results and Discussion}

\section{Effect of land configuration on growth characters}

The effect of land configuration on number of tillers per plant, plant height, number of leaves plant $^{-1}$, leaf area plant ${ }^{-1}$ and plant dry matter plant $^{-1}$ was statistically significant during both the years of investigation. Planting of turmeric on broad bed furrow recorded the maximum number of tillers plant ${ }^{-1}$ (1.78 and 1.86), plant height $(98.29$ and $101.71 \mathrm{~cm})$, number of leaves plant ${ }^{-1}$ (9.80 and 10.99), leaf area plant ${ }^{-}$ ${ }^{1}$ (44.70 and $48.77 \mathrm{dm}^{2}$ ) and plant dry matter plant $^{-1}$ (78.05 and 85.64g) compared to that ridges and furrow method during the year 2014 and 2015 respectively (Table1).

The present findings of number of tillers per plant are in consonance with the previous observation of Leva et al., (2013a) in turmeric. Better growth and greater partitioning in 
groundnut genotypes when grown on broad bed as compared to flat bed sowing was also observed by Talwar et al., (2002).

\section{Effect of irrigation levels on growth characters}

The differences among the irrigation levels treatments were significant during 2014 and 2015. Irrigation level $40 \mathrm{~mm}$ CPE recorded significantly more number of tillers plant ${ }^{-1}$ (2.25 and 2.33), plant height (100.23 and $104.52 \mathrm{~cm})$, number of leaves plant ${ }^{-1}(10.02$ and 11.45), leaf area plant ${ }^{-1}$ (46.95 and 50.07 $\mathrm{dm}^{2}$ ) and dry matter plant ${ }^{-1}(81.01$ and $90.31 \mathrm{~g})$ over $60 \mathrm{~mm}$ and $80 \mathrm{~mm}$ CPE irrigation levels in the year 2014 and 2015 (Table1).

Similar results were observed by Manjunathgoud et al., (2002), and Akamine et al., (2007) in turmeric. Singh (2010c) reported that at harvest number of leaves per plant were significantly higher in the crop grown at 1.25 IW: CPE irrigation regime as compared to 1.00, 0.75 and 0.50 IW: CPE in potato.

\section{Effect of nutrient management on growth characters}

Treatments of nutrient management significantly influenced the number of tillers plant $^{-1}$, plant height, number of functional leaves plant ${ }^{-1}$, leaf area plant ${ }^{-1}$, and dry matter plant $^{-1}$ during 2014 and 2015. The highest number of tillers plant ${ }^{-1}$ (2.00 and 2.11), plant height $(97.57$ and $101.51 \mathrm{~cm})$, number of functional leaves plant ${ }^{-1}$ (9.88 and 11.37), leaf area plant $\left(45.34\right.$ and $\left.50.76 \mathrm{dm}^{2}\right)$ and dry matter plant ${ }^{-1}(79.55$ and $85.28 \mathrm{~g})$ were recorded with $100 \%$ RDF $+25 \%$ RDN through vermicompost compared to other treatments (Table 1). Since, the number of tillers is an important yield attribute in turmeric, it would influence the yield and mother rhizomes as reported by Kamal and Yousuf (2012) and Singh (2015) in ginger.
Similar results in plant height were also noticed in turmeric by Tripathi et al., (2014), Singh S.P. (2013), Padmadevi et al., (2012), Parmeet et al., (2012), Manhas et al., (2011), Grima et al., (2008), Hikaru et al., (2007), Ram and Singh (2007) and Meenakshi et al., (1999).

\section{Interaction effect on growth characters}

From the data placed at Table 4, it was evident that number of tillers plant $^{-1}$, plant height plant $^{-1}$, number of functional leaves plant ${ }^{-1}$, leaf area plant $^{-1}$ and plant dry matter plant $^{-1}$ were affected significantly due to interaction between irrigation level $40 \mathrm{~mm}$ CPE with $100 \%$ RDF $+25 \%$ RDN through vermicompost.

Treatment combination of irrigation level 40 mm CPE with 100\% RDF +25 \% RDN through vermicompost recorded maximum number of tillers plant ${ }^{-1}$ (3.00 and 3.00), plant height plant $^{-1}(101.96$ and $107.47 \mathrm{~cm})$, number of functional leaves plant ${ }^{-1}$ (10.80 and 12.57), leaf area plant ${ }^{-1}$ (48.82 and $\left.54.36 \mathrm{dm}^{2}\right)$ and plant dry matter plant $^{-1}$ (89.95 and $95.41 \mathrm{~g}$ ) and was significantly superior over rest of the treatment combinations during 2014 and 2015 (Table 4).

Maximum number of tillers is in line with the findings of Tripathi et al., (2014). Similar finding was reported by Sandeep Kumar Tripathi et al., (2019). Anonymous (2013 a.) also found increase in leaf area of turmeric due to combination of nutrient management with irrigation.

\section{Effect of land configuration on yield}

Significant differences were noticed for yield, yield attributes and economics of turmeric due to the land configuration.

The number of mother rhizomes plant ${ }^{-1}$, 
weight of mother rhizomes plant $^{-1}$, fresh mother rhizomes $\mathrm{ha}^{-1}$ (q), yield of fresh primary fingers $\mathrm{ha}^{-1}$, fresh secondary fingers yield, total yield of turmeric and yield of cured fingers of turmeric were significantly influenced due to land configuration. Broad bed furrow method of planting recorded significantly highest number of mother rhizomes plant ${ }^{-1}$. (1.79, 1.78 and 1.78), mother rhizome weight $(67.30,78.60$ and $72.95 \mathrm{~g}$ plant $^{-1)}$, yields of fresh mother rhizomes (66.47, 77.63 and $72.05 \mathrm{q} \mathrm{ha}^{-1}$ ), fresh primary finger yield (119.97, 142.24 and $130.96 \mathrm{q} \mathrm{ha}^{-}$ $\left.{ }^{1}\right), \quad$ fresh secondary fingers yield $\left(38.48,47.71\right.$ and $\left.43.23 \mathrm{q} \mathrm{ha}^{-1}\right)$, total fresh rhizomes yield of turmeric (227.43,270.93 and $249.18 \mathrm{q} \mathrm{ha}^{-1}$ ) and yield of cured fingers ha ${ }^{1}\left(30.08,40.20\right.$ and $\left.35.14 \mathrm{q} \mathrm{ha}^{-1}\right)$ over the ridges and furrow planting during the year 2014 and 2015 respectively (Table 2 and 3). The results corroborate the earlier findings of Gill et al., (2009).

Haque et al., (2002) in garlic, observed significant improvement in growth attributes as well as yield of crops under similar kind of land configurations. Jayapaul et al., (1996) and Ardeshna et al., (2013) revealed similar results in their investigations regarding yields of fresh mother rhizomes.

The results corroborate the earlier findings of Ramachandran and Muthuswami (1984) and Amzad et al., (2005) who recorded markedly higher yield of turmeric when planted on broad bed furrow.

\section{Effect of irrigation levels on yield}

Irrigation levels produced significant effect on the number of mother rhizomes plant-1, weight of mother rhizomes plant ${ }^{-1}$, yield of fresh mother rhizomes ha ${ }^{-1}$, yield of fresh primary fingers $\mathrm{ha}^{-1}$, yield of fresh secondary fingers, total fresh rhizomes yield and yield of cured fingers of turmeric during individual years.
Frequent irrigation recorded maximum number of mother rhizomes plant- $(2.25,2.38$ and 2.31), weight of mother rhizomes plant ${ }^{-1}$ (89.87, 102.80 and $96.33 \mathrm{~g}$ ), yield of fresh mother rhizomes ha ${ }^{-1}(88.76,101.53$ and 95.14 $\mathrm{q} \mathrm{ha}{ }^{-1}$ ), yield of fresh primary fingers $\mathrm{ha}^{-1}$ $\left(128.86,157.61\right.$ and $\left.143.24 \mathrm{q} \mathrm{ha}^{-1}\right)$, Yield of fresh secondary fingers (45.42, 54.64 and $50.03 \mathrm{q} \mathrm{ha}^{-1}$ ), total fresh rhizomes yield of turmeric $\left(266.33,317.69\right.$ and $\left.292.01 \mathrm{q} \mathrm{ha}^{-1}\right)$ and yield of cured fingers (34.36,46.61 and $40.48 \mathrm{q} \mathrm{ha}^{-1}$ ) in irrigation level $40 \mathrm{~mm}$ CPE, compared to other irrigation level treatments (Table 2 and 3).

The yield of fresh mother rhizomes results are in accordance to those reported by Singh et al., (1998). The yield of fresh primary fingers results are in conformity with Verma et al., (2003). Rathod et al., (2010) in turmeric also found the increase in yield under more frequent irrigations $(40 \mathrm{~mm})$ might be due to its favourable effect on growth and yield contributing characters viz., plant height, number and weight of rhizomes plant ${ }^{-1}$. Mahey et al., (1986) observed similar type of results wherein, irrigation scheduled at $40 \mathrm{~mm}$ evaporation rate produced maximum rhizome yield over its increasing evaporation rate scheduled, as the farmers practice with more frequent irrigations which puts optimum moisture in the root zone resulting in more rhizome yield.

\section{Effect of nutrient management on yield}

Treatments of nutrient management significantly influenced the number of mother rhizomes plant ${ }^{-1}$, weight of mother rhizomes plant $^{-1}$, yield of fresh mother rhizomes $\mathrm{ha}^{-1}$, yield of fresh primary fingers $\mathrm{ha}^{-1}$, fresh secondary finger yield, total fresh rhizomes yield of turmeric and yield of cured fingers during the experimentation. The highest number of mother rhizomes plant ${ }^{-1}(2.00,2.00$ and 2.00), weight of mother rhizomes plant ${ }^{-1}$ 
(80.04, 91.89 and $85.97 \mathrm{~g}$ ), yield of fresh mother rhizomes per $\mathrm{ha}^{-1}(79.05,90.75$ and $84.90 \mathrm{q} \mathrm{ha}^{-1}$ ), yield of fresh primary fingers ha 1 (126.13,154.32 and $\left.140.23 \mathrm{q} \mathrm{ha}^{-1}\right)$, fresh secondary finger yield (42.21, 51.79 and 47.00 $\mathrm{q} \mathrm{ha}^{-1}$ ), the total fresh rhizomes yield of turmeric $\left(250.49,300.57\right.$ and $\left.275.53 \mathrm{q} \mathrm{ha}^{-1}\right)$ and yield of cured fingers $(33.54,45.24$ and $39.39 \mathrm{q} \mathrm{ha}^{-1}$ ) were recorded with $100 \% \mathrm{RDF}+$ $25 \%$ RDN through vermicompost as compared to others treatments (Table 2 and 3 ). Similar type of results was also reported by Sarkar et al., (2011) for number of mother rhizomes plant ${ }^{-1}$. Mannikeri (2006) also reported increase in yield of turmeric when organic is used in combination with inorganic fertilizers.

Sanwal (2007) and Sarma et al., (2015) recorded the maximum weight of mother, primary and secondary rhizomes and yield.

Venkatesha et al., (1998) in turmeric and Majumdar et al., (2003) in ginger also recorded increase in yield due to application of vermicompost with inorganic fertilizers.

Singh (2015) recorded highest rhizome yield in treatment having $100 \% \mathrm{NPK}+\mathrm{FYM}+$ poultry manure + vermicompost + wheat straw in ginger. Higher dry rhizome recovery in turmeric with organic ammendments was also reported by Rao et al., (2005) and Sanwal et al., (2007).

These results are in agreement with Majumdar et al., (2002), Manjunathgoud et al.,(2002), Kandiannan and Chandaragiri (2006), Krishnamoorthy et al., (2015) and Leva et al., (2013b).

\section{Interaction effect on yield}

The number of mother rhizomes plant ${ }^{-1}(3.00$, 3.00 and 3.00), weight of mother rhizomes plant $^{-1}(123.47,136.32$ and 129.89 g),yield of fresh mother rhizomes $\mathrm{ha}^{-1}(121.95,134.63$ and $128.29 \mathrm{q})$, yield of fresh primary fingers ha ${ }^{-}$ ${ }^{1}\left(145.46,179.03\right.$ and $\left.162.25 \mathrm{q} \mathrm{ha}^{-1}\right)$, yield of fresh secondary fingers $\mathrm{ha}^{-1}(55.68,65.88$ and $60.78 \mathrm{q})$, total fresh rhizomes yield of turmeric $\mathrm{ha}^{-1}(327.13,384.29$ and $355.71 \mathrm{q}$ ha-1) and yield of cured fingers $\mathrm{ha}^{-1}(45.17,60.71$ and $52.94 \mathrm{q} \mathrm{ha}^{-1}$ ) were significantly more in treatment combination $\mathrm{I}_{1} \times \mathrm{N}_{4}$ i.e. irrigation level $40 \mathrm{~mm}$ CPE with 100\% RDF +25\% RDN through vermicompost over other treatment combinations during the study period (Table 5 and 6).

Khan et al., (1999) reported that interaction of irrigation and nitrogen was significant in turmeric and recorded highest rhizome yield. The yield of fresh primary fingers results are in conformity with Tripathi Sandeep et al., (2015).

The results of total fresh rhizomes yield of turmeric are in accordance with the findings of Anonymous, 2013, Tripathi et al., (2014) and Samir Bhatti et al., (2019). The yield of cured fingers results are in agreement with Tripathi et al., (2014) and Sandeep Kumar Tripathi et al., (2019).

\section{Economic studies}

\section{Effect of land configuration}

The data revealed that there were significant differences in the gross monetary returns, net monetary returns and benefit: cost ratio among different treatments of land configuration.

Significantly higher gross monetary returns (421883, 467429 and $444656 \mathrm{Rs} \mathrm{ha}^{-1}$ ), net monetary returns (267742, 304161 and $285952 \mathrm{Rs} \mathrm{ha}^{-1}$ ) and higher benefit:cost ratio (2.71, 2.83 and 2.77) were recorded with broad bed method of planting as compare to ridges and furrow method (Table 3 ). 
Table.1 Growth attributes influenced by land configuration, irrigation levels and nutrient management during 2014 and 2015

\begin{tabular}{|c|c|c|c|c|c|c|c|c|c|c|}
\hline \multirow[t]{2}{*}{ Treatment/Year } & \multicolumn{2}{|c|}{ Number of tillers plant ${ }^{-1}$} & \multicolumn{2}{|c|}{$\begin{array}{l}\text { Plant height plant }{ }^{-1} \\
(\mathrm{~cm}) \text { at } 180 \text { DAP }\end{array}$} & \multicolumn{2}{|c|}{$\begin{array}{l}\text { Number of functional } \\
\text { leaves plant }{ }^{-1} \text { at } 180 \\
\text { DAP }\end{array}$} & \multicolumn{2}{|c|}{$\begin{array}{l}\text { Leaf area plant } \\
\left(\mathrm{dm}^{2}\right) \text { at } 180 \\
\text { DAP }\end{array}$} & \multicolumn{2}{|c|}{$\begin{array}{l}\text { Dry matter plant }{ }^{-1}(g) \text { a } \\
\text { harvest }\end{array}$} \\
\hline & 2014-2015 & 2015-2016 & $\begin{array}{l}2014- \\
2015\end{array}$ & 2015-2016 & 2014-2015 & 2015-2016 & 2014-2015 & 2015-2016 & 2014-2015 & 2015-2016 \\
\hline \multicolumn{11}{|l|}{ I) Main plot treatments } \\
\hline \multicolumn{11}{|l|}{ a) Land configuration } \\
\hline L1: Ridges and furrow & 1.64 & 1.67 & 94.92 & 98.55 & 9.32 & 10.57 & 43.86 & 46.82 & 73.03 & $\mathbf{7 8 . 7 3}$ \\
\hline L2: Broad bed furrow & 1.78 & 1.86 & 98.29 & 101.71 & 9.80 & 10.99 & 44.70 & 48.77 & 78.05 & 85.64 \\
\hline S. E. $(\mathbf{m}) \pm$ & 0.03 & 0.06 & 0.25 & 0.30 & 0.13 & 0.13 & 0.15 & 0.30 & 0.92 & 0.94 \\
\hline C. D. at $5 \%$ & 0.11 & 0.18 & 0.79 & 0.93 & 0.41 & 0.42 & 0.49 & 0.96 & 2.91 & 2.96 \\
\hline \multicolumn{11}{|l|}{ b) Irrigation levels } \\
\hline I1: 40 mm CPE & 2.25 & 2.33 & 100.23 & 104.52 & 10.02 & 11.45 & 46.95 & 50.07 & 81.01 & 90.31 \\
\hline I2: $60 \mathrm{~mm} \mathrm{CPE}$ & 1.79 & 1.83 & 96.55 & 99.70 & 9.73 & 10.67 & 44.22 & 46.80 & 74.88 & 81.00 \\
\hline I3: 80 mm CPE & 1.08 & 1.13 & 93.03 & 96.17 & 8.93 & 10.22 & 41.67 & 46.52 & 70.72 & 75.24 \\
\hline S. E. $(\mathbf{m}) \pm$ & 0.04 & 0.07 & 0.31 & 0.36 & 0.16 & 0.16 & 0.19 & 0.37 & 1.13 & 1.15 \\
\hline C. D. at 5\% & 0.13 & 0.22 & 0.97 & 1.14 & 0.51 & 0.51 & 0.60 & 1.17 & 3.56 & 3.62 \\
\hline \multicolumn{11}{|l|}{ II) Sub plot treatments } \\
\hline \multicolumn{11}{|l|}{ c) Nutrient management } \\
\hline N1: 100\% RDF (200:100:100 NPK kg/ha) & 1.61 & 1.54 & 96.74 & 99.88 & 9.45 & 10.67 & 44.13 & 47.99 & 75.01 & 81.60 \\
\hline $\begin{array}{l}\text { N2: } 75 \% \quad \text { RDF } \\
\text { vermicompost }\end{array}$ & 1.22 & 1.50 & 94.53 & 97.68 & 9.00 & 10.19 & 42.96 & 43.30 & 69.12 & 76.75 \\
\hline N3: $125 \%$ RDF & 2.00 & 1.94 & 97.56 & 101.45 & 9.86 & 10.99 & 44.70 & 49.19 & 78.47 & 85.11 \\
\hline $\begin{array}{l}\text { N4: } 100 \% \text { RDF }+25 \% \text { RDN through } \\
\text { vermicompost. }\end{array}$ & 2.00 & 2.11 & 97.57 & 101.51 & 9.88 & 11.37 & 45.34 & 50.76 & 79.55 & 85.28 \\
\hline S. E. $(\mathbf{m}) \pm$ & 0.07 & 0.11 & 0.45 & 0.48 & 0.20 & 0.26 & 0.32 & 0.55 & 1.32 & 1.40 \\
\hline C. D. at $5 \%$ & 0.21 & 0.32 & 1.29 & 1.37 & 0.58 & 0.74 & 0.93 & 1.57 & 3.79 & 4.01 \\
\hline \multicolumn{11}{|l|}{ Interaction effect } \\
\hline \multicolumn{11}{|l|}{ L x I } \\
\hline S. E. $(\mathbf{m}) \pm$ & 0.05 & 0.10 & 0.43 & 0.51 & 0.22 & 0.23 & 0.37 & 0.74 & 1.59 & 1.62 \\
\hline C. D. at $5 \%$ & NS & NS & NS & NS & NS & NS & NS & NS & NS & NS \\
\hline \multicolumn{11}{|l|}{$L \times N$} \\
\hline S. E. $(\mathrm{m}) \pm$ & 0.10 & 0.15 & 0.63 & 0.67 & 0.28 & 0.36 & 0.64 & 0.09 & 1.86 & 1.97 \\
\hline C. D. at 5\% & NS & NS & NS & NS & NS & NS & NS & NS & NS & NS \\
\hline \multicolumn{11}{|l|}{ I x N } \\
\hline S. E. $(\mathbf{m}) \pm$ & 0.12 & 0.19 & 0.77 & 0.82 & 0.35 & 0.44 & 0.79 & 1.33 & 2.28 & 2.41 \\
\hline C. D. at $5 \%$ & 0.36 & 0.55 & 2.22 & 2.37 & 1.009 & 1.27 & 1.60 & 2.71 & 6.56 & 6.93 \\
\hline \multicolumn{11}{|l|}{$L \times I \times N$} \\
\hline S. E. $(\mathrm{m}) \pm$ & 0.18 & 0.27 & 1.09 & 1.17 & 0.49 & 0.62 & 1.11 & 1.89 & 3.23 & 3.42 \\
\hline C. D. at $5 \%$ & NS & NS & NS & NS & NS & NS & NS & NS & NS & NS \\
\hline GM & 1.71 & 1.76 & 96.60 & 100.13 & 9.56 & 10.78 & 44.28 & 47.79 & 75.54 & 82.18 \\
\hline
\end{tabular}


Table.2 Yield attributes influenced by land configuration, irrigation levels and nutrient management during 2014 and 2015

\begin{tabular}{|c|c|c|c|c|c|c|c|c|c|c|c|c|c|c|c|}
\hline \multirow[t]{2}{*}{ Treatment / Year } & \multicolumn{3}{|c|}{$\begin{array}{l}\text { Number of mother rhizomes } \\
\text { plant }^{-1}\end{array}$} & \multicolumn{3}{|c|}{$\begin{array}{l}\text { Weight of mother rhizomes } \\
\text { plant }^{-1}(g)\end{array}$} & \multicolumn{3}{|c|}{$\begin{array}{l}\text { Yield of fresh mother rhizomes } \\
\left(q^{-1} h^{-1}\right)\end{array}$} & \multicolumn{3}{|c|}{$\begin{array}{l}\text { Yield of fresh primary fingers } \\
\left(q_{\text {ha }^{-1}}\right)\end{array}$} & \multicolumn{3}{|c|}{$\begin{array}{l}\text { Yield of fresh secondar } \\
\text { fingers } \\
\left(q^{-1} a^{-1}\right)\end{array}$} \\
\hline & $\begin{array}{l}2014- \\
2015\end{array}$ & $\begin{array}{l}2015- \\
2016\end{array}$ & $\begin{array}{l}\text { Pooled } \\
\text { mean }\end{array}$ & $\begin{array}{l}2014- \\
2015\end{array}$ & $\begin{array}{l}2015- \\
2016\end{array}$ & $\begin{array}{l}\text { Pooled } \\
\text { mean }\end{array}$ & $\begin{array}{l}2014- \\
2015\end{array}$ & $\begin{array}{l}2015- \\
2016\end{array}$ & $\begin{array}{l}\text { Pooled } \\
\text { mean }\end{array}$ & $\begin{array}{l}2014- \\
2015\end{array}$ & $\begin{array}{l}2015- \\
2016\end{array}$ & $\begin{array}{l}\text { Pooled } \\
\text { mean }\end{array}$ & $2014-$ & $\begin{array}{l}2015- \\
2016\end{array}$ & $\begin{array}{l}\text { Pooled } \\
\text { mean }\end{array}$ \\
\hline \multicolumn{16}{|l|}{ I) Main plot treatments } \\
\hline \multicolumn{16}{|l|}{ a) Land configuration } \\
\hline $\mathbf{L}_{1}$ : Ridges and furrow & 1.64 & 1.64 & 1.64 & 57.77 & 70.21 & 63.99 & 57.06 & 69.34 & 63.20 & 111.13 & 129.57 & 120.35 & 33.90 & 42.21 & 38.05 \\
\hline $\mathrm{L}_{2}$ : Broad bed furrow & 1.79 & 1.78 & 1.78 & 67.30 & 78.60 & 72.95 & 66.47 & 77.63 & 72.05 & 119.97 & 142.24 & 130.96 & 38.48 & 47.71 & 43.23 \\
\hline S. E. $(\mathbf{m}) \pm$ & 0.03 & 0.03 & 0.03 & 1.28 & 1.37 & 0.86 & 1.26 & 1.35 & 0.85 & 1.73 & 2.19 & 1.62 & 0.85 & 0.79 & 0.70 \\
\hline C. D. at 5\% & 0.11 & 0.10 & 0.10 & 4.03 & 4.31 & 2.49 & 3.98 & 4.26 & 2.46 & 4.94 & 6.26 & 4.72 & 2.67 & 2.48 & 2.05 \\
\hline \multicolumn{16}{|l|}{ b) Irrigation levels } \\
\hline $\mathrm{I}_{1}: 40 \mathrm{~mm}$ CPE & 2.25 & 2.38 & 2.31 & 89.87 & 102.80 & 96.33 & 88.76 & 101.53 & 95.14 & 128.86 & 157.61 & 143.24 & 45.42 & 54.64 & 50.03 \\
\hline$I_{2}: 60 \mathrm{~mm} \mathrm{CPE}$ & 1.79 & 1.75 & 1.77 & 60.87 & 72.22 & 66.54 & 60.12 & 71.52 & 65.82 & 120.20 & 141.26 & 130.73 & 37.75 & 46.04 & 41.89 \\
\hline$I_{3}: 80 \mathrm{~mm} \mathrm{CPE}$ & 1.08 & 1.00 & 1.04 & 36.86 & 48.21 & 42.54 & 36.41 & 47.61 & 42.01 & 97.14 & 108.86 & 103.00 & 25.41 & 34.19 & 29.08 \\
\hline S. E. $(\mathrm{m}) \pm$ & 0.04 & 0.04 & 0.04 & 1.57 & 1.68 & 1.05 & 1.55 & 1.65 & 1.04 & 2.12 & 2.68 & 1.98 & 1.04 & 0.96 & 0.86 \\
\hline C. D. at 5\% & 0.13 & 0.12 & 0.12 & 4.94 & 5.28 & 3.05 & 4.88 & 5.21 & 3.02 & 6.06 & 7.66 & 5.78 & 3.26 & 3.04 & 2.51 \\
\hline CV \% & - & & & & & & 12.28 & 11.03 & 10.60 & 8.98 & 9.65 & 10.93 & 14.03 & 10.50 & 14.71 \\
\hline \multicolumn{16}{|l|}{ II) Sub plot treatments } \\
\hline \multicolumn{16}{|l|}{ c) Nutrient management } \\
\hline $\begin{array}{l}\mathrm{N}_{1}: 100 \% \text { RDF (200:100:100 NPK } \\
\mathrm{kg} / \mathrm{ha})\end{array}$ & 1.61 & 1.50 & 1.56 & 54.01 & 65.35 & 59.68 & 53.34 & 64.54 & 58.94 & 113.21 & 129.84 & 121.53 & 34.57 & 42.14 & 38.36 \\
\hline $\begin{array}{l}\mathrm{N}_{2}: 75 \% \mathrm{RDF}+25 \% \text { RDN through } \\
\text { vermicompost }\end{array}$ & 1.22 & 1.44 & 1.33 & 46.18 & 57.53 & 51.86 & 45.61 & 56.82 & 51.21 & 103.65 & 120.17 & 111.91 & 32.42 & 40.72 & 36.57 \\
\hline$N_{3}: 125 \%$ RDF & 2.00 & 1.89 & 1.94 & 69.91 & 82.86 & 76.39 & 69.05 & 81.84 & 75.44 & 118.61 & 139.31 & 128.96 & 35.56 & 45.19 & 40.37 \\
\hline $\begin{array}{l}\mathrm{N}_{4}: 100 \% \text { RDF }+25 \% \\
\text { through vermicompost. }\end{array}$ & 2.00 & 2.00 & 2.00 & 80.04 & 91.89 & 85.97 & 79.05 & 90.75 & 84.90 & 126.13 & 154.32 & 140.23 & 42.21 & 51.79 & 47.00 \\
\hline S. E. $(m) \pm$ & 0.07 & 0.08 & 0.06 & 2.09 & 2.61 & 1.50 & 2.06 & 2.57 & 1.48 & 2.51 & 2.91 & 1.93 & 1.29 & 1.27 & 1.13 \\
\hline C. D. at 5\% & 0.21 & 0.22 & 0.18 & 5.98 & 7.47 & 4.22 & 5.91 & 7.38 & 4.17 & 7.19 & 8.33 & 5.44 & 3.70 & 3.63 & 3.17 \\
\hline CV \% & & & & & & & 14.15 & 14.85 & 13.16 & 9.21 & 9.07 & 9.23 & 15.11 & 11.95 & 16.63 \\
\hline \multicolumn{16}{|l|}{ Interaction effect } \\
\hline \multicolumn{16}{|l|}{ Lx I } \\
\hline S. E. $(\mathbf{m}) \pm$ & 0.05 & 0.05 & 0.05 & 2.21 & 2.36 & 1.48 & 2.18 & 2.34 & 1.46 & 2.99 & 3.78 & 2.80 & 1.46 & 1.36 & 1.22 \\
\hline C. D. at $5 \%$ & NS & NS & NS & NS & NS & NS & NS & NS & NS & NS & NS & NS & NS & NS & NS \\
\hline \multicolumn{16}{|l|}{$\mathbf{L} \times \mathbf{N}$} \\
\hline S. E. $(\mathrm{m}) \pm$ & 0.10 & 0.10 & 0.09 & 2.94 & 3.68 & 2.12 & 2.91 & 3.63 & 2.09 & 3.54 & 4.10 & 2.73 & 1.82 & 1.79 & 1.59 \\
\hline C. D. at 5\% & NS & NS & NS & NS & NS & NS & NS & NS & NS & NS & NS & NS & NS & NS & NS \\
\hline \multicolumn{16}{|l|}{ I x N } \\
\hline S. E. $(\mathrm{m}) \pm$ & 0.12 & 0.13 & 0.11 & 3.61 & 4.51 & 2.60 & 3.56 & 4.45 & 2.5 & 4.33 & 5.03 & 3.35 & 2.23 & 2.19 & 1.95 \\
\hline C. D. at $5 \%$ & 0.36 & 0.37 & 0.31 & 10.35 & 12.94 & 7.30 & 10.23 & 12.78 & 7.21 & 12.44 & 14.43 & 9.41 & 6.40 & 6.29 & 5.48 \\
\hline \multicolumn{16}{|l|}{ L x I x N } \\
\hline S. E. $(\mathrm{m}) \pm$ & 0.18 & 0.18 & 0.15 & 5.10 & 6.38 & 3.67 & 5.04 & 6.30 & 3.63 & 6.13 & 7.11 & 4.73 & 3.15 & 3.10 & 2.75 \\
\hline C. D. at 5\% & NS & NS & NS & NS & NS & NS & NS & NS & NS & NS & NS & NS & NS & NS & NS \\
\hline GM & 1.69 & 1.71 & 1.70 & 62.54 & 74.41 & 68.47 & 61.76 & 73.49 & 67.63 & 115.40 & 135.91 & 125.66 & 36.19 & 44.96 & 40.64 \\
\hline
\end{tabular}


Table.3 Yield attributes influenced by land configuration, irrigation levels and nutrient management during 2014 and 2015.

\begin{tabular}{|c|c|c|c|c|c|c|c|c|c|c|c|c|c|c|c|}
\hline \multirow[t]{2}{*}{ Treatment / Year } & \multicolumn{3}{|c|}{$\begin{array}{l}\text { Total yield of turmeric (Mother } \\
\text { +Primary } \\
\text { Rhizomes) } \\
\left(\mathbf{q ~ h a}^{-1}\right)\end{array}$} & \multicolumn{3}{|c|}{$\begin{array}{l}\text { Yield of cured fingers } \\
\left(\mathrm{q} \mathrm{ha}^{-1}\right)\end{array}$} & \multicolumn{3}{|c|}{$\begin{array}{l}\text { Gross monetary Returns } \text { ha }^{-1} \\
\text { (Rs.) }\end{array}$} & \multicolumn{3}{|c|}{ Net monetary Returns ha ${ }^{-1}$ (Rs.) } & \multicolumn{3}{|c|}{ B:C Ratio } \\
\hline & $2014-$ & $\begin{array}{l}2015- \\
2016\end{array}$ & $\begin{array}{l}\text { Pooled } \\
\text { mean }\end{array}$ & $\begin{array}{l}2014- \\
2015\end{array}$ & 2015-2016 & $\begin{array}{l}\text { Pooled } \\
\text { mean }\end{array}$ & $\begin{array}{l}2014- \\
2015\end{array}$ & $\begin{array}{l}2015- \\
2016\end{array}$ & $\begin{array}{l}\text { Pooled } \\
\text { mean }\end{array}$ & $\begin{array}{l}2014- \\
2015\end{array}$ & $\begin{array}{l}2015- \\
2016\end{array}$ & $\begin{array}{l}\text { Pooled } \\
\text { mean }\end{array}$ & $2014-$ & $\begin{array}{l}2015- \\
2016\end{array}$ & $\begin{array}{l}\text { Pooled } \\
\text { mean }\end{array}$ \\
\hline \multicolumn{16}{|l|}{ I) Main plot treatments } \\
\hline \multicolumn{16}{|l|}{ a) Land configuration } \\
\hline $\mathrm{L}_{1}$ : Ridges and furrow & 204.61 & 244.14 & 224.38 & 26.81 & 35.36 & 31.08 & 370518 & 413778 & 392148 & 219319 & 254545 & 236932 & 2.43 & 2.56 & 2.50 \\
\hline$L_{2}:$ Broad bed furrow & 227.43 & 270.93 & 249.18 & 30.08 & 40.20 & 35.14 & 421883 & 467429 & 444656 & 267742 & 304161 & 285952 & 2.71 & 2.83 & 2.77 \\
\hline S. E. $(\mathbf{m}) \pm$ & 2.03 & 2.74 & 1.91 & 0.57 & 0.87 & 0.58 & 4579 & 7239 & 4069 & 4579 & 7239 & 3997 & -- & -- & -- \\
\hline C. D. at 5\% & 5.80 & 7.86 & 5.58 & 1.64 & 2.49 & 1.68 & 13114 & 20700 & 11851 & 13130 & 20791 & 11642 & -- & -- & -- \\
\hline \multicolumn{16}{|l|}{ b) Irrigation levels } \\
\hline$I_{1}: 40 \mathrm{~mm}$ CPE & 266.33 & 317.69 & 292.01 & 34.36 & 46.61 & 40.48 & 513961 & 570756 & 542359 & 355203 & 401079 & 378141 & 3.22 & 3.34 & 3.28 \\
\hline $\mathrm{I}_{2}: 60 \mathrm{~mm}$ CPE & 220.80 & 261.86 & 241.33 & 29.39 & 38.44 & 33.92 & 400123 & 439728 & 419925 & 246102 & 277062 & 261582 & 2.59 & 2.70 & 2.65 \\
\hline$I_{3}: 80 \mathrm{~mm}$ CPE & 160.80 & 193.04 & 176.99 & 21.59 & 28.28 & 24.93 & 274518 & 311327 & 292922 & 129287 & 159917 & 144602 & 1.89 & 2.06 & 1.97 \\
\hline S. E. $(\mathbf{m}) \pm$ & 2.48 & 3.35 & 2.35 & 0.70 & 1.06 & 0.71 & 5608 & 8866 & 4983 & 5608 & 8866 & 4895 & -- & -- & -- \\
\hline C. D. at 5\% & 8.00 & 9.61 & 6.83 & 2.01 & 3.04 & 2.06 & 16078 & 25359 & 14515 & 16061 & 25383 & 14259 & -- & -- & -- \\
\hline CV \% & 9.89 & 8.37 & 6.86 & 12.09 & 12.94 & 14.79 & 6.94 & 9.86 & 8.25 & 11.28 & 15.55 & 12.97 & -- & -- & -- \\
\hline \multicolumn{16}{|l|}{ II) Sub plot treatments } \\
\hline \multicolumn{16}{|l|}{ c) Nutrient management } \\
\hline $\begin{array}{l}\mathrm{N}_{1}: 100 \% \text { RDF }(200: 100: 100 \text { NPK } \\
\mathrm{kg} / \mathrm{ha})\end{array}$ & 203.64 & 239.48 & 221.56 & 26.80 & 35.00 & 31.00 & 361164 & 400697 & 380931 & 216795 & 248809 & 232802 & 2.49 & 2.61 & 2.55 \\
\hline $\begin{array}{l}\mathrm{N}_{2}: \mathbf{7 5 \%} \text { RDF }+25 \% \text { RDN through } \\
\text { vermicompost }\end{array}$ & 183.96 & 220.42 & 202.19 & 24.99 & 32.57 & 28.78 & 326439 & 363515 & 344977 & 171470 & 201072 & 186271 & 2.10 & 2.23 & 2.16 \\
\hline $\mathrm{N}_{3}: \mathbf{1 2 5} \%$ RDF & 226.00 & 269.66 & 247.83 & 28.46 & 38.10 & 33.28 & 414496 & 463698 & 439097 & 266705 & 306949 & 286827 & 2.78 & 2.92 & 2.85 \\
\hline $\begin{array}{l}\mathrm{N}_{4}: 100 \% \text { RDF }+25 \% \text { RDN through } \\
\text { vermicompost. }\end{array}$ & 250.49 & 300.57 & 275.53 & 33.54 & 45.24 & 39.39 & 482704 & 534504 & 508604 & 319153 & 360581 & 339867 & 2.91 & 3.03 & 2.97 \\
\hline S. E. $(m) \pm$ & 3.51 & 3.77 & 2.56 & 0.76 & 1.15 & 0.66 & 8063 & 9641 & 5819 & 8063 & 9641 & 5771 & -- & -- & -- \\
\hline C. D. at 5\% & 10.03 & 10.82 & 7.18 & 2.19 & 3.31 & 1.85 & 23126 & 27653 & 16351 & 23126 & 27653 & 16214 & -- & -- & -- \\
\hline CV \% & 8.64 & 7.21 & 6.47 & 11.38 & 12.94 & 11.92 & 8.63 & 9.28 & 8.34 & 14.05 & 14.64 & 13.24 & -- & -- & -- \\
\hline \multicolumn{16}{|l|}{ Interaction effect } \\
\hline \multicolumn{16}{|l|}{ L x I } \\
\hline S. E. $(\mathbf{m}) \pm$ & 3.51 & 4.73 & 3.31 & 0.99 & 1.50 & 1.00 & 7931 & 12540 & 7047 & 7931 & 12539 & 6923 & -- & -- & -- \\
\hline C. D. at 5\% & NS & NS & NS & NS & NS & NS & NS & NS & NS & NS & NS & NS & -- & -- & -- \\
\hline \multicolumn{16}{|l|}{$L \times N$} \\
\hline S. E. $(\mathbf{m}) \pm$ & 4.95 & 5.33 & 3.61 & 1.07 & 1.62 & 0.93 & 11403 & 13635 & 8230 & 11403 & 13634 & 8161 & -- & -- & -- \\
\hline C. D. at 5\% & NS & NS & NS & NS & NS & NS & NS & NS & NS & NS & NS & NS & -- & -- & -- \\
\hline \multicolumn{16}{|l|}{ I x N } \\
\hline S. E. $(\mathbf{m}) \pm$ & 6.07 & 6.53 & 4.42 & 1.32 & 1.99 & 1.13 & 13966 & 16699 & 10080 & 13966 & 16699 & 9995 & --- & -- & -- \\
\hline C. D. at $5 \%$ & 17.41 & 18.74 & 12.43 & 3.79 & 5.72 & 3.20 & 40056 & 47896 & 28321 & 40056 & 47896 & 28084 & --- & -- & -- \\
\hline \multicolumn{16}{|l|}{ L x I x N } \\
\hline S. E. $(\mathbf{m}) \pm$ & 8.58 & 9.24 & 6.26 & 1.86 & 2.82 & 4.52 & 19750 & 23616 & 14255 & 19750 & 23616 & 14136 & -- & -- & -- \\
\hline C. D. at 5\% & NS & NS & NS & NS & NS & NS & NS & NS & NS & NS & NS & NS & -- & -- & -- \\
\hline GM & 216.02 & 257.53 & 236.78 & 28.45 & 37.78 & 33.11 & 396201 & 440604 & 418402 & 243531 & 279353 & 261442 & 2.57 & 2.70 & 2.63 \\
\hline
\end{tabular}


Table.4 Growth characters influenced by irrigation levels x nutrient management during 2014 and 2015.

\begin{tabular}{|c|c|c|c|c|c|c|c|c|c|c|c|c|c|c|c|c|c|c|c|c|}
\hline \multirow{2}{*}{$\begin{array}{l}\text { Irrigation } \\
\text { levels / } \\
\text { Nutrient } \\
\text { management }\end{array}$} & \multicolumn{4}{|c|}{$\underset{1}{\text { Number of tillers plant }}{ }^{-}$} & \multicolumn{4}{|c|}{ Plant height plant ${ }^{-1}(\mathrm{~cm})$} & \multicolumn{4}{|c|}{$\begin{array}{l}\text { Number of functional leaves } \\
\text { plant }^{-1}\end{array}$} & \multicolumn{4}{|c|}{ Leaf areaplant ${ }^{-1}\left(\mathrm{dm}^{2}\right)$} & \multicolumn{4}{|c|}{ Dry matter plant ${ }^{-1}(g)$} \\
\hline & $\mathbf{N}_{1}$ & $\mathbf{N}_{2}$ & $\mathbf{N}_{3}$ & $\mathbf{N}_{4}$ & $\mathbf{N}_{1}$ & $\mathbf{N}_{2}$ & $\mathbf{N}_{3}$ & $\mathbf{N}_{4}$ & $\mathbf{N}_{1}$ & $\mathbf{N}_{2}$ & $\mathbf{N}_{3}$ & $\mathbf{N}_{4}$ & $\mathbf{N}_{1}$ & $\mathbf{N}_{2}$ & $\mathbf{N}_{3}$ & $\mathbf{N}_{4}$ & $\mathbf{N}_{1}$ & $\mathbf{N}_{2}$ & $\mathbf{N}_{3}$ & $\mathbf{N}_{4}$ \\
\hline $\begin{array}{l}\mathrm{I}_{1}: 40 \mathrm{~mm} \\
\mathrm{CPE}\end{array}$ & 2.00 & 1.50 & 2.80 & 3.00 & 100.33 & 97.98 & 100.63 & 101.96 & 9.87 & 9.43 & 10.77 & 10.80 & 46.03 & 45.60 & 47.36 & 48.82 & 80.85 & 72.15 & 87.11 & 89.95 \\
\hline $\begin{array}{l}\mathrm{I}_{2}: \quad 60 \mathrm{~mm} \\
\mathrm{CPE}\end{array}$ & 1.70 & 1.20 & 2.00 & 2.00 & 96.25 & 94.39 & 97.24 & 98.32 & 9.47 & 8.93 & 9.93 & 10.10 & 44.15 & 43.91 & 44.29 & 44.52 & 71.93 & 69.08 & 77.48 & 77.96 \\
\hline $\begin{array}{l}\mathbf{I}_{3}: 80 \mathrm{~mm} \\
\mathrm{CPE}\end{array}$ & 1.00 & 1.00 & 1.20 & 1.20 & 91.56 & 91.23 & 94.51 & 94.81 & 8.70 & 8.63 & 8.93 & 9.13 & 42.20 & 39.37 & 42.45 & 42.67 & 70.33 & 66.13 & 71.21 & 72.25 \\
\hline S. E. $(\mathrm{m}) \pm$ & \multicolumn{4}{|l|}{0.12} & \multicolumn{4}{|l|}{0.77} & \multicolumn{4}{|l|}{0.35} & \multicolumn{4}{|l|}{0.55} & \multicolumn{4}{|l|}{2.28} \\
\hline C. D. at $5 \%$ & \multicolumn{4}{|c|}{0.36} & \multicolumn{4}{|l|}{2.22} & \multicolumn{4}{|l|}{1.00} & \multicolumn{4}{|l|}{1.60} & \multicolumn{4}{|c|}{6.56} \\
\hline & \multicolumn{4}{|c|}{ 2015-16 } & \multicolumn{4}{|c|}{ 2015-16 } & \multicolumn{4}{|c|}{ 2015-16 } & \multicolumn{4}{|c|}{ 2015-16 } & \multicolumn{4}{|c|}{$2015-16$} \\
\hline $\begin{array}{l}\mathrm{I}_{1}: 40 \mathrm{~mm} \\
\mathrm{CPE}\end{array}$ & 2.00 & 2.00 & 2.80 & 3.00 & 103.48 & 101.13 & 106.00 & 107.47 & 10.83 & 10.20 & 12.50 & 12.57 & 48.70 & 47.28 & 50.12 & 54.36 & 91.08 & 79.53 & 95.22 & 95.41 \\
\hline $\begin{array}{l}\mathrm{I}_{2}: \quad 60 \mathrm{~mm} \\
\mathrm{CPE}\end{array}$ & 1.50 & 1.50 & 1.80 & 2.00 & 99.40 & 97.53 & 100.38 & 101.47 & 10.53 & 9.93 & 10.90 & 11.00 & 48.50 & 41.33 & 49.09 & 49.20 & 78.38 & 75.79 & 84.28 & 85.56 \\
\hline $\begin{array}{l}\mathbf{I}_{3}: 80 \mathrm{~mm} \\
\mathrm{CPE}\end{array}$ & 1.00 & 1.00 & 1.20 & 1.30 & 94.70 & 94.37 & 97.66 & 97.95 & 10.33 & 9.57 & 10.43 & 10.53 & 46.77 & 41.28 & 48.07 & 48.83 & 74.91 & 74.54 & 75.32 & 76.15 \\
\hline S. E. $(\mathrm{m}) \pm$ & \multicolumn{4}{|l|}{0.19} & \multicolumn{4}{|l|}{0.82} & \multicolumn{4}{|l|}{0.44} & \multicolumn{4}{|l|}{0.94} & \multicolumn{4}{|l|}{2.41} \\
\hline C. D. at $5 \%$ & \multicolumn{4}{|l|}{0.55} & 2.37 & & & & 1.27 & & & & 2.71 & & & & 6.93 & & & \\
\hline
\end{tabular}

$\mathrm{N}_{1}: 100 \%$ RDF (200:100:100 NPK kg/ha), $\mathrm{N}_{2}: 75 \%$ RDF +25\% RDN through vermicompost, $\mathrm{N}_{3}: 125 \%$ RDF and $\mathrm{N}_{4}: 100 \%$ RDF $+25 \%$ RDN through vermicompost. 
Table.5 Yield characters influenced by irrigation levels x nutrient management during 2014 and 2015.

\begin{tabular}{|c|c|c|c|c|c|c|c|c|c|c|c|c|c|c|c|c|c|c|c|c|}
\hline \multirow{2}{*}{$\begin{array}{l}\text { Irrigation } \\
\text { levels / } \\
\text { Nutrient } \\
\text { management }\end{array}$} & \multicolumn{4}{|c|}{$\begin{array}{l}\text { Number of mother } \\
\text { rhizomes plant }^{-1}\end{array}$} & \multicolumn{4}{|c|}{$\begin{array}{l}\text { Weight of mother rhizomes } \\
\text { plant }^{-1}(\mathrm{~g})\end{array}$} & \multicolumn{4}{|c|}{$\begin{array}{l}\text { Yield of fresh mother rhizomes } \\
\text { ha }^{-1}(q)\end{array}$} & \multicolumn{4}{|c|}{$\begin{array}{l}\text { Yield of fresh primary fingers ha } \\
{ }^{1}(q)\end{array}$} & \multicolumn{4}{|c|}{$\begin{array}{l}\text { Yield of fresh secondary } \\
\text { fingers } \mathrm{ha}^{-1}(\mathrm{q})\end{array}$} \\
\hline & $\mathbf{N}_{1}$ & $\mathbf{N}_{2}$ & $\mathbf{N}_{3}$ & $\mathbf{N}_{4}$ & $\mathrm{~N}_{1}$ & $\mathbf{N}_{2}$ & $\mathbf{N}_{3}$ & $\mathbf{N}_{4}$ & $\mathrm{~N}_{1}$ & $\mathbf{N}_{2}$ & $\mathbf{N}_{3}$ & $\mathbf{N}_{4}$ & $\mathrm{~N}_{1}$ & $\mathbf{N}_{2}$ & $\mathbf{N}_{3}$ & $\mathbf{N}_{4}$ & $\mathrm{~N}_{1}$ & $\mathbf{N}_{2}$ & $\mathbf{N}_{3}$ & $\mathbf{N}_{4}$ \\
\hline & \multicolumn{4}{|c|}{ 2014-2015 } & \multicolumn{4}{|c|}{ 2014-2015 } & \multicolumn{4}{|c|}{ 2014-2015 } & \multicolumn{4}{|c|}{ 2014-2015 } & \multicolumn{4}{|c|}{ 2014-2015 } \\
\hline $\begin{array}{l}\mathrm{I}_{1}: 40 \mathrm{~mm} \\
\mathrm{CPE}\end{array}$ & 2.00 & 1.50 & 2.83 & 3.00 & 78.02 & 56.91 & 101.10 & 123.47 & 77.06 & 56.20 & 99.85 & 121.95 & 120.07 & 118.96 & 130.96 & 145.46 & 44.00 & 37.27 & 44.71 & 55.68 \\
\hline $\begin{array}{l}\mathrm{I}_{2}: \quad 60 \mathrm{~mm} \\
\text { CPE }\end{array}$ & 1.67 & 1.17 & 2.00 & 2.00 & 48.30 & 47.04 & 71.92 & 76.22 & 47.71 & 46.46 & 71.03 & 75.28 & 124.38 & 100.35 & 127.10 & 128.99 & 35.18 & 34.69 & 37.48 & 43.63 \\
\hline $\begin{array}{l}\mathrm{I}_{3}: \quad 80 \mathrm{~mm} \\
\text { CPE }\end{array}$ & 1.00 & 1.00 & 1.17 & 1.17 & 35.69 & 34.61 & 36.72 & 40.44 & 35.25 & 34.18 & 36.27 & 39.94 & 95.19 & 91.65 & 97.76 & 103.95 & 23.82 & 22.51 & 27.31 & 27.98 \\
\hline S. E. $(\mathrm{m}) \pm$ & \multicolumn{4}{|l|}{0.12} & \multicolumn{4}{|l|}{3.61} & \multicolumn{4}{|l|}{3.56} & \multicolumn{4}{|l|}{4.33} & \multicolumn{4}{|l|}{2.23} \\
\hline C. D. at $5 \%$ & \multicolumn{4}{|c|}{0.36} & \multicolumn{4}{|c|}{10.35} & \multicolumn{4}{|c|}{10.23} & \multicolumn{4}{|l|}{12.44} & \multicolumn{4}{|c|}{6.40} \\
\hline & \multicolumn{4}{|c|}{ 2015-16 } & \multicolumn{4}{|c|}{ 2015-16 } & \multicolumn{4}{|c|}{ 2015-16 } & \multicolumn{4}{|l|}{ 2015-16 } & \multicolumn{4}{|c|}{ 2015-16 } \\
\hline $\begin{array}{l}\mathrm{I}_{1}: 40 \mathrm{~mm} \\
\mathrm{CPE}\end{array}$ & 2.00 & 1.67 & 2.83 & 3.00 & 89.37 & 68.25 & 117.25 & 136.32 & 88.26 & 67.41 & 115.80 & 134.63 & 148.42 & 136.11 & 166.86 & 179.03 & 50.82 & 45.56 & 56.29 & 65.88 \\
\hline $\begin{array}{l}\mathrm{I}_{2}: \quad 60 \mathrm{~mm} \\
\text { CPE }\end{array}$ & 1.67 & 1.50 & 1.83 & 2.00 & 59.65 & 58.38 & 83.27 & 87.57 & 58.91 & 57.66 & 82.24 & 86.48 & 139.28 & 119.10 & 146.97 & 159.69 & 43.48 & 42.99 & 45.78 & 51.93 \\
\hline $\begin{array}{l}\mathrm{I}_{3}: \quad 80 \mathrm{~mm} \\
\text { CPE }\end{array}$ & 1.00 & 1.00 & 1.00 & 1.00 & 47.03 & 45.95 & 48.07 & 51.78 & 46.45 & 45.38 & 47.47 & 51.14 & 101.83 & 105.28 & 104.09 & 124.23 & 32.12 & 30.81 & 36.28 & 37.56 \\
\hline S. E. $(\mathrm{m}) \pm$ & \multicolumn{4}{|l|}{0.13} & \multicolumn{4}{|l|}{4.51} & \multicolumn{4}{|l|}{4.45} & 5.03 & & & & 2.19 & & & \\
\hline C. D. at $5 \%$ & 0.37 & & & & 12.94 & & & & 12.78 & & & & 14.43 & & & & 6.29 & & & \\
\hline & Poole & d mear & & & Pooled & mean & & & Pooled & mean & & & Pooled I & mean & & & Poolec & mean & & \\
\hline $\begin{array}{l}\mathrm{I}_{1}: 40 \mathrm{~mm} \\
\text { CPE }\end{array}$ & 2.00 & 1.58 & 2.83 & 3.00 & 83.69 & 62.58 & 109.17 & 129.89 & 82.66 & 61.81 & 107.83 & 128.29 & 134.24 & 127.54 & 148.91 & 162.25 & 47.41 & 41.41 & 50.50 & 60.78 \\
\hline $\begin{array}{l}\mathrm{I}_{2}: 60 \mathrm{~mm} \\
\mathrm{CPE}\end{array}$ & 1.67 & 1.33 & 1.92 & 2.00 & 53.98 & 52.71 & 77.59 & 81.89 & 53.31 & 52.06 & 76.64 & 80.88 & 131.83 & 109.72 & 137.03 & 144.34 & 39.33 & 38.84 & 41.63 & 47.78 \\
\hline $\begin{array}{l}\mathrm{I}_{3}: 80 \mathrm{~mm} \\
\mathrm{CPE}\end{array}$ & 1.00 & 1.00 & 1.08 & 1.08 & 41.36 & 40.28 & 42.39 & 46.11 & 40.85 & 39.78 & 41.87 & 45.54 & 98.51 & 98.47 & 100.93 & 114.09 & 27.97 & 26.66 & 31.79 & 32.77 \\
\hline S. E. (m) \pm & 0.11 & & & & 2.60 & & & & 2.56 & & & & 3.35 & & & & 1.95 & & & \\
\hline C. D. at $5 \%$ & 0.31 & & & & 7.30 & & & & 7.21 & & & & 9.41 & & & & 5.48 & & & \\
\hline
\end{tabular}

$\mathrm{N}_{1}: 100 \%$ RDF (200:100:100 NPK kg/ha), $\mathrm{N}_{2}: 75 \%$ RDF +25\% RDN through vermicompost, $\mathrm{N}_{3}: 125 \%$ RDF and $\mathrm{N}_{4}: 100 \%$ RDF $+25 \%$ RDN through vermicompost. 
Table.6 Yield characters and economics influenced by irrigation levels x nutrient management during 2014 and 2015

\begin{tabular}{|c|c|c|c|c|c|c|c|c|c|c|c|c|c|c|c|c|}
\hline \multirow{2}{*}{$\begin{array}{l}\text { Irrigation } \\
\text { levels / } \\
\text { Nutrient } \\
\text { management }\end{array}$} & \multicolumn{4}{|c|}{$\begin{array}{l}\text { Total fresh rhizomes yield of } \\
\text { turmeric } \text { ha }^{-1}(q)\end{array}$} & \multicolumn{4}{|c|}{$\begin{array}{l}\text { Yield of cured fingers } \mathrm{ha}^{-1} \\
\text { (q) }\end{array}$} & \multicolumn{4}{|c|}{ Gross monetary returns (Rs ha- $\left.{ }^{1}\right)$} & \multicolumn{4}{|c|}{ Net monetary returns ( $R s$ ha- $\left.^{1}\right)$} \\
\hline & $\mathbf{N}_{1}$ & $\mathbf{N}_{2}$ & $\mathbf{N}_{3}$ & $\mathbf{N}_{4}$ & $\mathbf{N}_{1}$ & $\mathbf{N}_{2}$ & $\mathbf{N}_{3}$ & $\mathbf{N}_{4}$ & $\mathbf{N}_{1}$ & $\mathbf{N}_{2}$ & $\mathbf{N}_{3}$ & $\mathbf{N}_{4}$ & $\mathbf{N}_{1}$ & $\mathbf{N}_{2}$ & $\mathbf{N}_{3}$ & $\mathbf{N}_{4}$ \\
\hline & \multicolumn{4}{|c|}{ 2014-2015 } & \multicolumn{4}{|c|}{ 2014-2015 } & \multicolumn{4}{|c|}{ 2014-2015 } & \multicolumn{4}{|c|}{ 2014-2015 } \\
\hline $\begin{array}{l}\mathrm{I}_{1}: 40 \mathrm{~mm} \\
\mathrm{CPE}\end{array}$ & 244.86 & 215.09 & 278.24 & 327.13 & 30.99 & 28.32 & 32.96 & 45.17 & 456075 & 381212 & 529755 & 688804 & 307220 & 220749 & 376146 & 516698 \\
\hline $\begin{array}{l}\mathrm{I}_{2}: \quad 60 \mathrm{~mm} \\
\text { CPE }\end{array}$ & 209.86 & 186.59 & 235.74 & 251.00 & 28.43 & 26.33 & 30.13 & 32.67 & 360933 & 339985 & 433679 & 465897 & 213812 & 184734 & 284111 & 301753 \\
\hline $\begin{array}{l}\text { I }_{3}: 80 \mathrm{~mm} \\
\text { CPE }\end{array}$ & 156.19 & 150.20 & 164.03 & 173.34 & 20.98 & 20.31 & 22.28 & 22.77 & 266486 & 258121 & 280055 & 293412 & 129355 & 108928 & 139859 & 139008 \\
\hline S. E. $(\mathrm{m}) \pm$ & \multicolumn{4}{|l|}{6.07} & \multicolumn{4}{|l|}{1.32} & \multicolumn{4}{|l|}{13966} & \multicolumn{4}{|l|}{13966} \\
\hline C. D. at $5 \%$ & \multicolumn{4}{|l|}{17.41} & \multicolumn{4}{|c|}{3.79} & \multicolumn{4}{|l|}{40056} & \multicolumn{4}{|l|}{40056} \\
\hline & \multicolumn{4}{|c|}{ 2015-16 } & \multicolumn{4}{|c|}{ 2015-16 } & \multicolumn{4}{|l|}{ 2015-16 } & \multicolumn{4}{|l|}{ 2015-16 } \\
\hline $\begin{array}{l}\mathrm{I}_{1}: 40 \mathrm{~mm} \\
\mathrm{CPE}\end{array}$ & 291.09 & 252.20 & 343.19 & 384.29 & 42.61 & 36.91 & 46.20 & 60.71 & 510428 & 419527 & 603674 & 749399 & 351134 & 251287 & 436871 & 565026 \\
\hline $\begin{array}{l}\text { I }_{2}: 60 \mathrm{~mm} \\
\text { CPE }\end{array}$ & 244.70 & 225.32 & 275.61 & 301.84 & 36.47 & 34.70 & 39.09 & 43.51 & 395249 & 380147 & 471438 & 512080 & 240890 & 216759 & 313452 & 337151 \\
\hline $\begin{array}{l}\mathrm{I}_{3}: 80 \mathrm{~mm} \\
\mathrm{CPE}\end{array}$ & 182.65 & 183.74 & 190.19 & 215.59 & 26.51 & 26.09 & 29.01 & 31.50 & 296416 & 290874 & 315984 & 342034 & 154404 & 135171 & 170528 & 179568 \\
\hline S. E. $(\mathrm{m}) \pm$ & \multicolumn{4}{|l|}{6.53} & \multicolumn{4}{|l|}{1.99} & \multicolumn{4}{|l|}{16699} & \multicolumn{4}{|l|}{16699} \\
\hline C. D. at $5 \%$ & \multicolumn{4}{|c|}{18.74} & \multicolumn{4}{|c|}{5.72} & \multicolumn{4}{|l|}{47896} & \multicolumn{4}{|l|}{47896} \\
\hline & \multicolumn{4}{|c|}{ Pooled mean } & \multicolumn{4}{|c|}{ Pooled mean } & Pooled I & nean & & & Pooled I & nean & & \\
\hline $\begin{array}{l}\mathrm{I}_{1}: 40 \mathrm{~mm} \\
\mathrm{CPE}\end{array}$ & 267.98 & 233.64 & 310.72 & 355.71 & 36.80 & 32.62 & 39.58 & 52.94 & 483252 & 400369 & 566715 & 719101 & 329177 & 236018 & 406508 & 540862 \\
\hline $\begin{array}{l}\mathrm{I}_{2}: 60 \mathrm{~mm} \\
\mathrm{CPE}\end{array}$ & 227.28 & 205.95 & 255.67 & 276.42 & 32.45 & 30.52 & 34.61 & 38.09 & 378091 & 360066 & 452558 & 488989 & 227351 & 200747 & 298781 & 319452 \\
\hline $\begin{array}{l}\mathrm{I}_{3}: 80 \mathrm{~mm} \\
\mathrm{CPE}\end{array}$ & 169.42 & 166.97 & 177.11 & 194.47 & 23.75 & 23.20 & 25.65 & 27.13 & 281451 & 274497 & 298019 & 317723 & 141879 & 122050 & 155193 & 159288 \\
\hline S. E. $(\mathrm{m}) \pm$ & 4.42 & & & & 1.13 & & & & 10080 & & & & 9995 & & & \\
\hline C. D. at $5 \%$ & 12.43 & & & & 3.23 & & & & 28321 & & & & 28084 & & & \\
\hline
\end{tabular}

$\mathrm{N}_{1}: 100 \%$ RDF (200:100:100 NPK kg/ha), $\mathrm{N}_{2}: 75 \%$ RDF +25\% RDN through vermicompost, $\mathrm{N}_{3}: 125 \%$ RDF and $\mathrm{N}_{4}: 100 \%$ RDF $+25 \%$ RDN through vermicompost. 
The results regarding land configuration also lend support to the earlier findings of Sinare B.T.et al., (2016) and Hanamant M. Halli and S.S. Angadi (2018). The net monetary return findings confirm with the earlier findings of Patil et al., (1991), Bheemappa et al., (1994) in groundnut, Shete et al., (2010) and Joshi et al., (2018). Sudhakar et al., (2017) in safflower and Hanamant and Angadi (2018) in turmeric recorded higher benefit:cost ratio from crops raised on broad bed furrow land configurations.

\section{Effect of irrigation levels}

The data in table 3 showed significant effect of irrigation levels treatment on gross monetary returns and net monetary returns per hectare and benefit: cost ratio.

Highest gross monetary returns (513961, 570756 and $542359 \mathrm{Rs} \mathrm{ha}^{-1}$ ), net monetary returns per hectare $(355203,401079$ and 378141Rs $\mathrm{ha}^{-1}$ ) and benefit: cost ratio (3.22,3.34 and 3.28) were recorded significantly in irrigation level $40 \mathrm{~mm} \mathrm{CPE}$ as compared to others irrigation levels. The gross monetary returns results are in conformity with the earlier findings of Nisha Nandle (2018) in onion. Benefit: cost ratio results have also been reported by Chakraborty et al., (2010) and Chauhan (2015).

\section{Effect of nutrient management}

Nutrient management treatments significantly influenced the gross monetary returns and net monetary returns per hectare and benefit: cost ratio during the experimentation (Table 3 ).

Significantly the highest gross monetary return (482704, 534504 and $\left.508604 \mathrm{Rs} \mathrm{ha}^{-1}\right)$, net monetary return $\mathrm{ha}^{-1}(319153,360581$ and 339867Rs ha ${ }^{-1}$ ) and benefit: cost ratio (2.91, 3.03 and 2.97) were recorded in treatment $\mathrm{N} 4$ $(100 \% \mathrm{RDF}+25 \% \mathrm{RDN}$ through vermicompost) over all other treatments during the study period and in pooled data respectively (Table 3 ). Similar results have been reported by other investigators Rana et al., (1993) and Sharma et al., (2003) regarding gross monetary returns.

\section{Interaction effect}

Data presented in Table 6 showed that gross monetary returns and net monetary returns ha1 was affected significantly due to interaction between irrigation level and nutrient management during both the years and in pooled analysis.

The gross monetary returns and net monetary returns ha-1 was noticed higher in $\mathrm{I}_{1} \mathrm{XN}_{4}$ treatment combination and recorded significantly highest gross monetary returns ha-1 $\left(688804,749399\right.$ and 719101Rs ha $\left.{ }^{-1}\right)$ and net monetary returns ha-1(516698,565026 and 540862 $\mathrm{Rs} \mathrm{ha}^{-1}$ ) during the course of study and in pooled data respectively over the other treatments (Table 6).

Combination of irrigation treatment with nutrient management resulted higher yield of turmeric and recorded higher gross monetary returns. Similar results was reported by Singh et al., (2002) in potato regarding net monetary returns ha ${ }^{-1}$.

Economic efficiency and the viability of crop cultivation are mainly the outcome of the yield of crop with higher management costs. Higher crop productivity with lesser cost of cultivation could result in better economic parameters like net returns and B: C ratio. Patra et al., (1998) and Singh (2015) in ginger showed that soil application of organic manures i.e., farm yard manures \& vermicompost, recommended dose of chemical fertilizers and integrated nutrient management i.e., organic and inorganic fertilizers showed significant effect with 
higher benefit: cost ratio. Based on the findings of two years investigation, it can be concluded that for higher production and net profit from turmeric (var. PDKV- Waigaon) can be secured by planting on broad bed furrow with irrigation level of $40 \mathrm{~mm}$ CPE and application of $100 \% \mathrm{RDF}+25 \% \mathrm{RDN}$ through vermicompost in heavy black (clay) soil of Vidarbha region under irrigated condition.

\section{References}

Akamine, H., Hossain, M.A., Ishimine, Y., Yogi, K., Hokama, K., Iraha, Y and Aniya Y. 2007. Effects of application of $\mathrm{N}, \mathrm{P}$ and $\mathrm{K}$ alone and in combination on growth, yield and curcumin content of turmeric (Curcuma longa L.). Plant Production Science. $10: 151-154$

Amzad Hossain, Yukio Ishimine, Keiji Motomura and Hikaru Akamine (2005) Effects of planting pattern and planting distance on growth and yield of turmeric (Curcuma longa L.). Plant Prod. Sci., 8 (1): $95-105$

Anonymous $\left(2013^{\mathrm{a}}\right)$ All India Coordinated Research Project on Water Management, Directorate of water management. Annual report (2012 - 2013), Bhubaneswar : pp 78

Anonymous (2015) National Horticulture Board. www.nhb.gov.in

Ardeshna R.B., M.K. Arvadia, R.G. Patil and N.G. Savania (2013) Effect of land configuration and soil conditioners on growth and yield of turmeric (Curcuma longa). Indian Journal of Agronomy, 58 (3): 412- 415.

Chakraborty H, Sethi Ranu, Pothula Srinivasa, Mandal K.G., Singandhupe Ramlal and Kumar Ashwani (2010) Effect of irrigation regimes and nitrogen levels on herbage and oil yield, oil quality, nutrient uptake and economics of Iemon grass (Cymbopogon flexuosus) in a sandy loam soil of Orissa. Indian Journal of Agricultural Sciences, 80: 713-718

Chauhan S. (2015) Fertigation management in turmeric (Curcuma longa L.) based cropping system. PhD. (Agri.) thesis. Acharya N.G. Ranga Agricultural University, Rajendranagar Hyderabad

Grima H. and Kindie, T. (2008) The effects of seed rhizome size on the growth, yield and economic return of ginger (Zingiber officinale Rosc.). Asian Journal of Plant Sciences, 7(2): 213-17

Hanamant M. Halli and S.S. Angadi (2018) Influence of land configuration on rain water use efficiency, yield and economics of cowpea (Vigna unguiculata L.) in maize-cowpea sequence cropping under rainfed condition of Northern Transitional Zone. Legume Research, 4 (2):127 -131

Haque S., Sattar A. and Pramanik H. R.(2002) Land configuration and varietal effects on yield contributing traits and yield of garlic. Pakistan J. Bio. Sci., 5 (10) : 1024-1027

Hikaru A., Hossain A., Ishimine Y., Yogi K., Hokama Y., Iraha Y. and Yoko A.(2007) Effects of application of N, P and K alone or in combination on growth, yield and curcumin content of turmeric (Curcuma longa L.). Plant Production Science, 10 (1): 151-154

Jayapaul P., Udayakumar B.,Devasagayam M. M., Pandian B. J., Palchamy A. and Balkrishnan A. (1996) Effect of land configuration methods, irrigation regimes and soil moisture conservation amendments on soybean (Glycine max (L.) Merr.) yield and quality characters. Crop Res., 10 (3) : 253-257

Joshi J.R.,V.M. Patel, H.L. Barad, S.M. Macwan and Javid Ehsas (2018) Effect of Land Configuration and Fertilizer Management Practices on Growth, Yield and Yield Attributes and Economics of Summer Cowpea (Vigna unguiculata L.) under South Gujarat Condition. Int.J.Curr.Microbiol.App.Sci., $\quad 7(1)$ : 1148-1155 
Kamal M.Z.U. and M. N. Yousuf (2012) Effect of Organic Manures on Growth, Rhizome Yield and Quality Attributes of Turmeric (Curcuma longa L.). The Agriculturists, 10 (1) : 16-22

Kandiannan K. and Chandaragir K. K. (2006) Influence of varieties, dates of planting, spacing and nitrogen levels on growth, yield and quality of turmeric (Curcuma longa). Indian J. Agric. Sci., 76 (7) : 432-434

Khan M. M and Shivashankar K., Krishna Manohar R., Sree Rama R and Kasiyanna (1999). Fertigation in horticultural crops. In: Proc. Advances in Micro Irrigation and Fertigation, June 21-30, Dharwad. pp: 181-197

Krishnamoorthy C., Soorianathasundaram K. and Mekala S.(2015) Effect of fertigation on fue, quality and economics of cultivation in turmeric (Curcuma longa L.) cv. BSR 2. International Journal of Agricultural Science and Research, 5 (1) : 67-72

Kumar S., Ram A. and Mandal G. (2007) Effect of differential irrigation regimes on potato (Solanum tuberosum) yield and post-harvest attributes. Indian Journal of Agricultural Sciences, 77 (6) : 366-368

Leva R. L, J. D. Thanki, D. D. Patel and T.U. Patel $\left(2013^{\mathrm{a}}\right)$ Effect of planting methods and N-K fertigation on growth and yield of turmeric (Curcuma longa L.) BIOINFOLET, 10 (3) : 811-813

Leva R. L., Thanki J. D., Patel D. D and Patel T. U. $\left(2013^{\mathrm{b}}\right)$ Growth and yield of turmeric (Curcuma longa L.) as influenced by planting methods and fertigation under vertisols of South Gujarat condition. Research on Crops, 14 (3): 964-967

Mahey R. K., Randhwa G. S and Gill S. R. S. (1986) Effect of irrigation and mulching on water conservation, growth and yield of turmeric. Indian Journal of Agronomy, 31 (1): 79-82

Majumdar B., Venkatesh M. S. and Kumar K.(2002) Effect of nitrogen and farmyard manure on yield and nutrient uptake of turmeric (Curcuma longa) and different forms of inorganic $\mathrm{N}$ build-up in an acidic Alfisol of Meghalaya. Indian J. Agril. Sci., 72 (9) : 528-531

Majumdar B., Venkatesh M.S., Kailash Kumar and Patiram (2003) Effect of N levels, FYM and mother rhizome removal on yield, nutrient uptake and quality of ginger (Zingiber officinale Rosc.) and different forms of $\mathrm{N}$ build up in an acidic Alfisol of Meghalaya. Crop Res. 25 (3): $478-483$

Manhas S.S., Gill B.S., Khajuria V. and Kumar S. (2011) Effect of planting material, mulch and farmyard manure on weed density, rhizome yield and quality of turmeric (Curcuma longa). Indian Journal of Agronomy, 56(4): 393-39.

Manjunathgoud B., Venkatesha J. and Bhagavantagoudra K.H.(2002). Studies on plant density and levels of NPK on growth, yield and quality of turmeric cv. Bangalore local. Mysore Journal of Agricultural Research, 36:31-35

Mannikeri.I. M. (2006) Studies on production technology of Turmeric. (Curcuma longa L.). Ph. D. Thesis. Department of Horticulture, University of Agriculture Sciences, Dharwad, Karnataka state, India

Meenakshi N., Sulikeri G.B., Ramakrishna V. and Hegde (1999). Effect of planting material and $\mathrm{P} \& \mathrm{~K}$ on plant growth of turmeric. Karnataka Journal of Agricultural Sciences, 14(1):194-196

Mohamed M A, Wahba H E, Ibrahim M E, Yousef A A.(2014) Effect of irrigation intervals on growth and chemical composition of some Curcuma spp. plants. Nusantara Bioscience, 6: 140-145

Nisha Nandle, R.K. Sharma, S.S. Kushwah and Roshan Gallani(2018) Effect of Irrigation Regimes and Varieties on Growth, Bulb Yield and Quality of Onion (Allium cepa L.). Int. J. Curr. Microbiol. App. Sci., 7 (5): 1104-1111

Padmadevi K., Jeeva Jothi L., Ponnuswami V., Durgavathi V. and Rijwana Parveen, I. (2012) Effect of different grades of rhizomes on growth and yield of 
turmeric (Curcuma longa L.). The Asian Journal of Horticulture, 7 (2) : 465-67

Patra A. P., Panda D., Patra B. C. and Karmarkar A. J. (1998) Effect of FYM, zinc and NPK fertilizers on yield components and yield of wheat after winter rice in West Bengal. J. Interacademicia, 2 (2) : 1-6

Panse V.G. and Sukhtame P.V.(1985) Statistical methods for agricultural workers. ICAR, New Delhi.

Parmeet S., Rather M. A., Badri Z.A., Jyothi S. and Alie B.A.(2012) Effect of root tuber weight and spacing on kala zeera (Bunium persicum) in Gurez valley of Kashmir. Indian Journal of Agronomy, 57 (3): $279-83$

Ram and Singh (2007) Influence of types of rhizomes and plant geometry on growth, yield of turmeric (Curcuma longa L.). Progressive Agriculture, 7 (1/2):110-12

Rana S. C., Rattan R. S., Faceria M. S. and Parmar Y. S. (1993) Varietal response to different levels of nitrogen and phosphorus for quality attributes in turmeric (Curcuma longa L.). Indian Cocoa Arecanut and Spices Journal, 17(1-2): 29-30

Rao A. M., Rao P. V., Reddy Y. N. and Reddy M. S. N. (2005) Effect of organic and inorganic manurial combinations on growh, yield and quality of turmeric (Curcuma longa L.). J. Plantation Crops, 33 (3): 198-205

Rathod S. D., Kamble B. M and Pawar V. P. (2010) Effect of irrigation and levels of fertilizer on yield of turmeric in vertisols irrigated through micro sprinkler. Advances in Plant Sciences, 23 (1): 201203

Samir Bhatti J.C. Sharma and Ridham Kakar (2019) Effect of Irrigation and Nitrogen Levels on Nutrient Uptake, Water Use Efficiency and Productivity of Onion (Allium cepa L.) in Himachal Pradesh. Int.J.Curr.Microbiol.App.Sci. 8 (2): 398408

Sandeep Kumar Tripathi, Babloo Sharma, R. Ray, Monika Devi and P.Mishra (2019)
Studies on growth and yield of turmeric under different irrigation and nutrient management strategies at West Bengal. Bulletin of Environment, Pharmacology and Life Sciences Bull. Env. Pharmacol. Life Sci., 8 (6) : 14-20

Sanwal S. K., Laxminarayana K., Yadav R. K., Rai N., Yadav D. S. and Bhuyan, M. (2007) Effect of organic manures on soil fertility, growth, physiology, yield and quality of turmeric. Indian J. Hort., 64 (4): 444 - 449

Sarkar A.,Sarkar S. and Zaman A.(2011) Growth and yield of potato as influenced by combination of organic manures and inorganic fertilizers. Potato Journal 38 (1): $78-80$

Sarkar V., Lawande K.E and Tripathi P.C.(2015) Effect of micro irrigation on growth, yield and water-use-efficiency of onion(Allium cepa) under western Maharashtra conditions. Indian Journal of Agricultural Sciences. 78 (7): 584 588

Sarma M., Phukon and Roopa Borgohain (2015) Effect of organic manure, vermicompost and neemcake on growth, yield and profitability of turmeric (Curcuma longa L.) variety- Megha Turmeric-1. Asian Journalof Bioscience, 10 (2): 133-137

Sharma D. P., Sharma T. R., Agrawal S. B. and Rawat A.(2003) Differential response of turmeric to organic and inorganic fertilizers. JNKVV Research Journal 37: 17-19

Sinare B.T., R.P. Andhale, H.P. Pardeshi and A.D. Tambe(2016) Effect of Different Land Configurations, Irrigation Regimes and Potassium Levels on Consumptive Use, Water Use Efficiency and Yield of Summer Groundnut (Arachis hypogaea L.). Indian Journal of Ecology, 43 (2): 615-618

Singh, V.B., N.P. Singh and B. Swer. (1998) Effect of potassium and nitrogen on yield and quality of turmeric (Curcuma longa). J. Potassium Res., 15 (1/4) : 8892

Singh N., Sood M. C. and Singh S.P. $\left(2010^{c}\right)$ 
Optimizing irrigation water and nutrient requirement of potato (Solanum tuberosum L.) under drip fertigation. Progressive Agriculture, 10 (1): 192-195

Singh S.P.(2013) Influence of Bio-fertilizers in Conjunction with Organic and Inorganic Fertilizer on Soil Properties and Productivity of Turmeric Curcuma longa L. Indian Journal of Ecology, 42 (1) : 170-173

Singh S.P.(2015) Nutrient supplementation through organic manures for growth and yield of ginger (Zingiber officinale Rose.) Journal of Eco-friendly Agriculture, 10 (1): 28-31

Singh N., Sood M.C. and Sharma R.C.(2002). Effect of irrigation levels, cultural practices and nitrogen application on potato production under drip and sprinkler methods of irrigation. Potato, Global Research and Development Proceedings of the Global Conference on Potato, New Delhi, India, 6-11 December, 2002, Volume-2, pp.923-925 Sudhakar C., C. Sudha Rani, S. N. C. V. L. Pushpavlli, T. Rajeshwar Reddy, M. V. Nageshkumar (2017) Studies on influenceof broad bed and furrow system in in-situ moisture conservation, yield and economics of safflower (Carthamus tinctorius L.) under receding soil moisture conditions. Life Sciences International Research Journal, 4 (1) : 54-57
Talwar H. S., Soni M. L., Beniwal R. K., Tomar R. K. and Niga, S. N. (2002) Evaluation of short-duration groundnut genotypes for the arid zone of northwest Rajasthan, India. Int. Arachis Newsletter, 22 : 15-18

Tripathi S. K., Ray R., Patra S. K. (2014) Effect of irrigation and nutrient levels on growth, yield attributes and yield of turmeric (Curcuma longa L.) under alluvial soils of West Bengal. Environment and Ecology, 32, (3A) : $1096-1099$

Tripathi Sandeep,Sharma Babloo, Meena Santosh, Ray Rapti and Patra,Sanmay (2015) Influence of Irrigation Schedules and Nutrient Sources on Yield, Quality, Water Use and Water Productivity of Turmeric under Alluvial Soils of West Bengal. Journal of Pure and Applied Microbiology, 9 : 3109-3114

Venkatesha J., Khan M. M. and Chandrappa H. (1998) Studies on uptake of NPK nutrients by turmeric cultivars. Journal of Maharashtra Agricultural University, 23: $12-14$

Verma S.U., Sanjeev Kumar Pal, S.K and Thakur R. (2003) Growth analysis of wheat cultivars under different seedlings dates and irrigation levels in Jharkhand. Indian Journal of Agronomy, 48(4): 282286

\section{How to cite this article:}

Dikey, H. H., V. M. Bhale, V. S. Kale and Wankhade, R. S. 2019. Effect of Land Configuration, Irrigation Level and Nutrient Management on Growth, Yield and Economics of Turmeric (Curcuma longa L.). Int.J.Curr.Microbiol.App.Sci. 8(09): 2306-2322. doi: https://doi.org/10.20546/ijcmas.2019.809.266 